\title{
Intermittent Catheterization: The Devil Is in the Details
}

\author{
Kathleen Christison, ${ }^{1, *}$ Matthias Walter, ${ }^{1, *}$ Jean-Jacques J.M. Wyndaele, ${ }^{2}$ Michael Kennelly, ${ }^{3}$ Thomas M. Kessler, \\ Vanessa K. Noonan, Nader Fallah, ${ }^{5,6}$ and Andrei V. Krassioukov ${ }^{1,7,8}$
}

\begin{abstract}
During the last few years, the international community debated urinary tract infection and re-use of catheters when managing neurogenic lower urinary tract dysfunction (NLUTD) among individuals with spinal cord injury (SCI). In this respect, the 2014 Cochrane review by Prieto and colleagues, "Intermittent catheterisation for long-term bladder management," became one of the leading documents that captured the minds and attention of clinicians around the world. Although numerous countries had switched to single-use catheters for management of NLUTD following SCI, the opinion that was expressed in the 2014 Cochrane review had a strong influence on healthcare providers and agencies to recommend re-use of catheters. However, many clinicians have expressed concern regarding the conclusions in the 2014 Cochrane review by Prieto and colleagues. We therefore conducted an independent appraisal of the data and analyses presented in the review. Our appraisal identified crucial discrepancies of data extraction and analyses within the review. In appraisal to that of Prieto and colleagues' review, our analysis revealed a trend to favor single over multiple use of catheters. After addressing our concerns to Cochrane's acting Editor-in-Chief, the most recent version of the 2014 Cochrane review was withdrawn from publication.
\end{abstract}

Keywords: Cochrane review; intermittent catheterization; neurogenic lower urinary tract dysfunction; spinal cord injury; urinary tract infection

\section{Introduction}

D URING THE LAST FEW YEARS, the international community has engaged in strong debates on urinary tract infection (UTI) and re-use of catheters during the management of neurogenic lower urinary tract dysfunction (NLUTD) among individuals with spinal cord injury (SCI). The most frequent complication of intermittent catheterization is UTI. ${ }^{1}$ There is no universally accepted definition of UTI in individuals with NLUTD, particularly in those with SCI. While UTI is an evolving term that varies between organizations, its potentially devastating effect is of no argument. UTI is costly to both the healthcare system and to individuals and can result in frequent hospitalization, sepsis, and even death. ${ }^{2}$
In this respect, the Cochrane 2014 systematic review "Intermittent catheterisation for long-term bladder management" by Prieto and colleagues, became one of the leading documents that captured the minds and attention of clinicians around the world.,

Although the authors did identify numerous limitations and risk for bias within the trials included in their review (p.8), they came to the conclusion that "there is still no convincing evidence that the incidence of UTI is affected by use of aseptic or clean technique, coated or uncoated catheters, single (sterile) or multiple-use (clean) catheters, self-catheterisation or catheterisation by others, or by any other strategy" (p.2). This has influenced clinicians' opinions and recommendations on intermittent catheterization over the last few years.

\footnotetext{
${ }^{1}$ International Collaboration on Repair Discoveries (ICORD), ${ }^{6}$ Division of Neurology, Department of Medicine, ${ }^{7}$ Division of Physical Medicine and Rehabilitation, Department of Medicine, Faculty of Medicine, University of British Columbia, Vancouver, British Columbia, Canada.

${ }^{2}$ Department of Urology, University of Antwerp, Antwerp, Belgium.

${ }^{3}$ Department of Urology, Carolinas Medical Center, Charlotte, North Carolina.

${ }^{4}$ Neuro-Urology, Spinal Cord Injury Center \& Research, University of Zürich, Balgrist University Hospital, Zürich, Switzerland.

${ }^{5}$ Rick Hansen Institute, Vancouver, British Columbia, Canada.

${ }^{8}$ G.F. Strong Rehabilitation Center, Vancouver, British Columbia, Canada.

*The first two authors contributed equally.

(c) Kathleen Christison et al. (2018); Published by Mary Ann Liebert, Inc. This Open Access article is distributed under the terms of the Creative Commons License (http://creativecommons.org/licenses/by/4.0), which permits unrestricted use, distribution, and reproduction in any medium, provided the original work is properly credited.
} 
However, upon closer inspection of the review, we are confident that this conclusion requires revision.

\section{Methods}

We completed a thorough appraisal of the 2014 Cochrane review by Prieto and colleagues, focusing on 1) correct data selection and extraction of all 31 trials included, that is, 13 randomized controlled trials (RCTs) and 18 cross-over RCTs (Table 1), 2) use of an up-todate definition of UTI, and 3) statistical appropriateness and correctness of all 39 analyses.

\section{Results}

We identified four main concerns with the 2014 Cochrane review by Prieto and colleagues:

\section{First concern (data selection)}

Of the 31 trials included in the 2014 Cochrane review, 2 were published only as conference abstracts (Table 1). Further, Prieto and colleagues reported "Eight of the 31 trials did not provide data in a format that could be used in meta-analysis" (p.8). However, 2 of these 8 trials (Table 1 ) did provide data that could have been included in meta-analysis.

\section{Second concern (data extraction)}

Upon close inspection five types of disagreements were observed:

1. Data were mislabeled;

2. Extracted data did not match data from original trial;

3. Data were extracted in a method not consistent with convention;

4. Data were in a form that could not be used in meta-analyses; and

5. Data were not originally extracted, although eligible (Supplementary Table 1 and Fig. 1; see online supplementary material at http://www.liebertpub.com).

For example, in "Analysis 2.2" data from six of the eight trials provided by Prieto and colleages were not consistent with originally published data. At times, it appears that the authors extracted data from an original trial correctly, but placed the data under an incorrect heading or the data were presented only partially, that is, one instead of both arms of the cross-over trial was reported.

\section{Third concern (symptomatic UTI definition)}

Although the review was published in 2014, the UTI definition was taken from an outdated 1992 National Institute on Disability

Table 1. Trials Included in the 2014 Cochrane Review $(N=31)$

\begin{tabular}{|c|c|c|c|c|}
\hline No. & Year & Author & Trial design & Journal, year, (month), volume, issue, pages \\
\hline 1 & 2013 & Chartier-Kastler et al. & Cross-over RCT & J. Urol. 2013 Sep;190(3):942-7. \\
\hline 2 & 2013 & Costa et al. & Cross-over RCT & Spinal Cord 2013 Oct;51(10):772-5. \\
\hline 3 & 2013 & Leek et al. & Cross-over $\mathrm{RCT}^{\mathbf{c}}$ & Neurourol. Urodyn. 2012;32(6):759-60. \\
\hline 4 & 2013 & Moore et al. & Cross-over $\mathrm{RCT}^{\mathbf{c}}$ & Neurourol. Urodyn. 2013;32(6):760-1. \\
\hline 5 & 2012 & Denys et al. & Cross-over $\mathrm{RCT}^{\mathrm{d}}$ & Spinal Cord 2012 Nov;50(11):853-8. \\
\hline 6 & 2011 & Cardenas et al. & RCT & PM R 2011 May;3(5):408-17. \\
\hline 7 & 2011 & Chartier-Kastler et al. & Cross-over RCT & Spinal Cord 2011 Jul;49(7):844-50. \\
\hline 8 & 2011 & Domurath et al. & Cross-over RCT & Spinal Cord $2011 \mathrm{Jul} ; 49(7): 817-21$. \\
\hline 9 & 2010 & Sarica et al. & Cross-over $\mathrm{RCT}^{\mathrm{a}, \mathrm{d}}$ & Eur. J. Phys. Rehabil. Med. 2010 Dec;46(4):473-9. \\
\hline 10 & 2009 & Cardenas and Hoffman & RCT & Arch. Phys. Med. Rehabil. 2009 Oct;90(10):1668-71. \\
\hline 11 & 2009 & Witjes et al. & RCT & J. Urol. 2009 Dec;182(6):2794-8. \\
\hline 12 & 2007 & Biering-Sorensen et al. & Cross-over RCT & Scand. J. Urol. Nephrol. 2007;41(4):341-5. \\
\hline 13 & 2006 & Leriche et al. & Cross-over RCT & Prog. Urol. 2006 Jun;16(3):347-51. \\
\hline 14 & 2006 & Moore et al. & RCT & Clin. Rehabil. 2006 Jun;20(6):461-8. \\
\hline 15 & 2005 & De Ridder et al. & RCT & Eur. Urol. 2005 Dec;48(6):991-5. \\
\hline 16 & 2005 & Taweesangsuksalul et al. & Cross-over $\mathrm{RCT}^{\mathrm{d}}$ & J. Thai Rehabil. Med. 2005;15(2):113-8. \\
\hline 17 & 2003 & Day et al. & RCT & Urol. Nurs. 2003 Apr;23(2):143-7, 158. \\
\hline 18 & 2003 & Vapnek et al. & RCT & J. Urol. 2003 Mar;169(3):994-8. \\
\hline 19 & 2002 & Fera et al. & $\mathrm{RCT}^{\mathrm{d}}$ & Braz. J. Urol. 2002;28(1):50-6. \\
\hline 20 & 2001 & Fader et al. & Cross-over $\mathrm{RCT}^{\mathrm{b}, \mathrm{d}}$ & BJU Int. 2001 Sep;88(4):373-7. \\
\hline 21 & 2001 & Giannantoni et al. & Cross-over $\mathrm{RCT}^{\mathrm{d}}$ & J. Urol. $2001 \mathrm{Jul} ; 166(1): 130-3$. \\
\hline 22 & 2001 & Mauroy et al. & Cross-over $\mathrm{RCT}^{\mathrm{a}, \mathrm{d}}$ & Ann. Urol. (Paris) 2001 Jul;35(4):223-8. \\
\hline 23 & 2001 & Pascoe and Clovis & Cross-over RCT ${ }^{\mathrm{d}}$ & Br. J. Nurs. 2001 Mar 8-21;10(5):325-9. \\
\hline 24 & 2001 & Schlager et al. & Cross-over RCT & Pediatrics 2001 Oct;108(4): E71. \\
\hline 25 & 1999 & Pachler and Frimodt-Moller & Cross-over RCT & BJU Int. 1999 May;83(7):767-9. \\
\hline 26 & 1997 & Prieto-Fingerhut et al. & RCT & Rehabil. Nurs. 1997 Nov-Dec;22(6):299-302. \\
\hline 27 & 1996 & Sutherland et al. & $\mathrm{RCT}$ & J. Urol. 1996 Dec;156(6):2041-3. \\
\hline 28 & 1995 & Duffy et al. & $\mathrm{RCT}$ & J. Am. Geriatr. Soc. 1995 Aug;43(8):865-70. \\
\hline 29 & 1993 & Moore et al. & Cross-over RCT & Rehabil. Nurs. 1993 Sep-Oct;18(5):306-9. \\
\hline 30 & 1993 & Quigley and Riggin & $\mathrm{RCT}$ & Rehabil. Nurs. 1993 Jan-Feb;18(1):26-9, 33. \\
\hline 31 & 1992 & King et al. & $\mathrm{RCT}$ & Arch. Phys. Med. Rehabil. 1992 Sep;73(9):798-802. \\
\hline
\end{tabular}

Overall, 31 trials, comprising 13 parallel group randomized controlled trials (RCTs) and 18 cross-over RCTs, were included for qualitative analysis in the 2014 Cochrane review. The crossover RCTs either had two $\left(n=15\right.$, unmarked), three $\left(n=2\right.$, marked $\left.^{\mathrm{a}}\right)$, or four arms $\left(n=1\right.$, marked $\left.{ }^{\mathrm{b}}\right)$.

${ }^{c}$ Reports were only available as congress or meeting abstracts.

${ }^{\mathrm{d}}$ Eight of 31 trials were classified as not providing data in a format that could be used in meta-analysis by Prieto and colleagues. However, two trials, i.e., \#9 (Sarica et al. 2010) and \#21 (Giannantoni et al. 2001) provided data that could have been included in meta-analysis. 
Table 2. Comparison of Definitions of Symptomatic Urinary Tract Infection

$(N I D R R)-1992^{5}$

- Bacteriuria ( $\geq 100$ bacteria/mL of urine) with tissue invasion and resultant tissue response with signs and/or symptoms.

- Signs and symptoms: Leukocytes in the urine generated by the mucosal lining; discomfort or pain over the kidney or bladder, or during urination; onset of urinary incontinence; fever; increased spasticity; autonomic hyperreflexia; cloudy urine with increased odor; malaise, lethargy, or sense of unease.
$(I D S A)-2009^{6}$

- CA-UTI in patients with indwelling urethral, indwelling suprapubic, or intermittent catheterization is defined by the presence of symptoms or signs compatible with UTI with no other identified source of infection along with $\geq 1000 \mathrm{cfu} / \mathrm{mL}$ of $\geq 1$ bacterial species in a single catheter urine specimen or in a midstream voided urine specimen from a patient whose urethral, suprapubic, or condom catheter has been removed within the previous $48 \mathrm{~h}$.

- Data are insufficient to recommend a specific quantitative count for defining CA-UTI in symptomatic men when specimens are collected by condom catheter.

- CA-ASB should not be screened for except in research studies evaluating interventions designed to reduce CA-ASB or CA-UTI and in selected clinical situations, such as in pregnant women.

- CA-ASB in patients with indwelling urethral, indwelling suprapubic, or intermittent catheterization is defined by the presence of $\geq 100,000 \mathrm{cfu} / \mathrm{mL}$ of $\geq 1$ bacterial species in a single catheter urine specimen in a patient without symptoms compatible with UTI.

- CA-ASB in a man with a condom catheter is defined by the presence of $\geq 100,000 \mathrm{cfu} / \mathrm{mL}$ of $\geq 1$ bacterial species in a single urine specimen from a freshly applied condom catheter in a patient without symptoms compatible with UTI.

- Signs and symptoms compatible with CA-UTI include new onset or worsening fever, rigors, altered mental status, malaise, or lethargy with no other identified cause; flank pain; costovertebral angle tenderness; acute hematuria; pelvic discomfort; and in those whose catheters have been removed, dysuria, urgent or frequent urination, or suprapubic pain or tenderness.

- In patients with spinal cord injury, increased spasticity, autonomic dysreflexia, or sense of unease are also compatible with CA-UTI.

- In the catheterized patient, pyuria is not diagnostic of CA-bacteriuria or CA-UTI. The presence or absence of odorous or cloudy urine alone should not be used to differentiate CAASB from CA-UTI or as an indication for urine culture or antimicrobial therapy.

- The presence, absence, or degree of pyuria should not be used to differentiate CA-ASB from CA-UTI.

- Pyuria accompanying CA-ASB should not be interpreted as an indication for antimicrobial treatment.

- The absence of pyuria in a symptomatic patient suggests a diagnosis other than CA-UTI.

CA-ASB, catheter-associated asymptomatic bacteriuria; CA-UTI, catheter-associated urinary tract infection; cfu/mL, colony-forming units per milliliter; IDSA, Infectious Diseases Society of America; NIDRR, National Institute on Disability and Rehabilitation Research; UTI, urinary tract infection.

FIG. 1. A new perspective on analyses 1.2, 2.2, and 3.2 of the 2014 Cochrane review. (A) Analysis 1.2: Aseptic versus other technique. All five trials (UTI defined according to Cochrane) from the original Cochrane meta-analysis were included. Applying the Mantel-Haenszel (M-H) method (fixed effect model) for meta-analysis did not show any significant difference $(p=0.608)$ between aseptic versus other technique with regards to the incidence of UTI. (B) Analysis 2.2: Single versus multiple use of catheters. Seven of eight trials (UTI defined according to Cochrane) from the original Cochrane meta-analysis were included. Moore et al. 2013 was excluded because of missing data. Applying the $\mathrm{M}-\mathrm{H}$ method (fixed effect model) for meta-analysis did not show any significant difference $(p=0.593)$ between single versus multiple use of catheters with regards to the incidence of UTI. (C) Analysis 3.2: Hydrophilic versus other catheters. Four of five trials (UTI defined according to Cochrane) from the original Cochrane meta-analysis were included. Moore et al. 2013 was excluded because of missing data. Applying the M-H method (fixed effect model) for metaanalysis did show a significant difference $(p=0.043)$ between hydrophilic versus other catheters with regards to the incidence of UTI. De Ridder et al. 2005 is the only trial providing significant evidence favoring hydrophilic over another type of catheter. This trial is also the only one with a high number of participants $(n=123)$ and long investigation period (12 months). The authors of the Cochrane review refrained from deriving a summary estimate because of the heterogeneity among the trials and attrition bias. However, we did not find an issue with heterogeneity (see results above). (D) Analysis 1.2: Aseptic versus other technique. After adjustment was made regarding the UTI definition (according to the Infectious Diseases Society of America [IDSA]), only two trials were included in this analysis. Applying the M-H method (fixed effect model) for meta-analysis did not show any significant difference $(p=0.866)$ between aseptic versus other technique with regards to the incidence of UTI. Given the small number of participants $(n=82)$ and unclear duration of investigation (between 4 weeks and a minimum of 7 weeks), no final conclusion can be drawn. (E) Analysis 2.2: Single versus multiple use of catheters. After adjustment was made regarding the UTI definition (according to the IDSA), only three trials were included in this analysis. Applying the M-H method (fixed effect model) for meta-analysis did not show any significant difference $(p=0.512)$ between single versus multiple use of catheters with regards to the incidence of UTI. Given the small number of participants $(n=140)$ and short duration of investigation (maximum 8 weeks), no final conclusion can be drawn. (F) Analysis 3.2: Hydrophilic versus other catheters. After adjustment was made regarding the UTI definition (according to the IDSA), only two trials were included in this analysis. Applying the M-H method (fixed effect model) for meta-analysis did not show any significant difference $(p=0.945)$ between hydrophilic versus other catheters with regards to the incidence of UTI. Given the small number of participants $(n=94)$ and short duration of the investigation (maximum 8 weeks), no final conclusion can be drawn. CI, confidence interval. 
A

Aseptic Other

Risk Ratio

Risk Ratio

Trial Events Total Events Total Weight M-H, Fixed, $95 \% \mathrm{Cl} \quad \mathrm{M}-\mathrm{H}, \mathrm{Fixed}, 95 \% \mathrm{Cl}$

\begin{tabular}{|c|c|c|c|c|c|c|c|}
\hline King 1992 & 3 & 23 & 5 & 23 & $11.3 \%$ & 0.60 & {$[0.16 ; 2.22]$} \\
\hline Duffy 1995 & 22 & 42 & 20 & 38 & $47.6 \%$ & 1.00 & {$[0.66 ; 1.51]$} \\
\hline Prieto-Fingerhut 1999 & 8 & 14 & 9 & 15 & $19.7 \%$ & 0.95 & {$[0.52 ; 1.76]$} \\
\hline Moore 2006 & 9 & 20 & 6 & 16 & $15.1 \%$ & 1.20 & {$[0.54 ; 2.66]$} \\
\hline Quigley 1993 & 0 & 11 & 2 & 9 & $6.2 \%$ & 0.17 & {$[0.01 ; 3.04]$} \\
\hline
\end{tabular}

42 42

Heterogeneity: $I^{2}=0 \%, \tau^{2}=0, H=1.00, Q=2.31, d f=4, p=0.678$

Test for overall effect: $Z=-0.51, p=0.608$

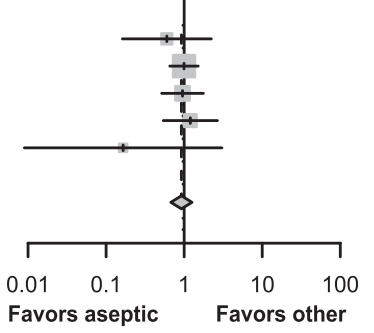

B

Single

Multiple

Risk Ratio

Risk Ratio

Trial Events Total Events Total Weight M-H, Fixed, $95 \% \mathrm{Cl}$

M-H, Fixed, $95 \%$ CI

Pachler 1999

Sutherland 1996

Leek 2013

Schlager 2001

Duffy 1995

King 1992

Prieto-Fingerhut 1999

Total $(95 \% \mathrm{Cl})$

Total events

$2.3 \% \quad 1.00 \quad[0.07 ; 15.30]$

$\begin{array}{rr}1 & 32 \\ 1 & 16 \\ 3 & 21 \\ 2 & 10 \\ 22 & 42 \\ 3 & 23 \\ 8 & 14\end{array}$

$\begin{array}{ll}1 & 32 \\ 1 & 14\end{array}$

$2.5 \% \quad 0.88 \quad[0.06 ; 12.73]$

$9.4 \% \quad 0.75 \quad[0.19 ; 2.95]$

$4.7 \% \quad 1.00 \quad[0.17 ; 5.77]$

$\begin{array}{rrrrr}2 & 10 & 4.7 \% & 1.00 & {[0.17 ; 5.77]} \\ 5 & 38 & 49.1 \% & 1.00 & {[0.66 ; 1.51]}\end{array}$

$\begin{array}{rr}2 & 10 \\ 20 & 38\end{array}$

$20.3 \% \quad 0.95 \quad[0.52 ; 1.76]$

Heterogeneity: $I^{2}=0 \%, \tau^{2}=0, \mathrm{H}=1.00, \mathrm{Q}=0.67, \mathrm{df}=6, p=0.995$

Test for overall effect: $Z=-0.53, p=0.593$

$100.0 \% \quad 0.91 \quad[0.66 ; 1.27]$ 42

C

Hydrophilic Other

Risk Ratio

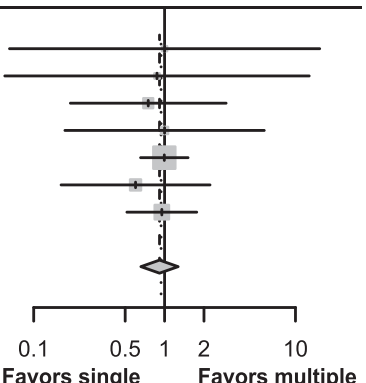

Trial Events Total Events Total Weight $\mathrm{M}-\mathrm{H}$, Fixed, $95 \% \mathrm{Cl}$

Risk Ratio

Cardenas 2009 Sutherland 1996 Pachler 1999 De Ridder 2005

$\begin{array}{rr}12 & 22 \\ 1 & 16 \\ 1 & 32 \\ 39 & 61\end{array}$

$\begin{array}{rr}14 & 23 \\ 1 & 1 \\ 1 & 32 \\ 51 & 62\end{array}$

$23 \quad 20.6 \% \quad 0.90 \quad[0.54 ; 1.48]$
$14 \quad 1.6 \% \quad 0.88[0.06 ; 12.73]$

Total $(95 \% \mathrm{Cl})$

131 53

131

$\begin{array}{llll}1.5 \% & 1.00 & {[0.07 ; 15.30]}\end{array}$

$76.3 \% \quad 0.78 \quad[0.62 ; 0.97]$

Total events 67

Heterogeneity: $I^{2}=0 \%, \tau^{2}=0, H=1.00, Q=0.3, d f=3, p=0.959$

Test for overall effect: $Z=-2.02, p=0.043$

$100.0 \% \quad 0.81 \quad[0.65 ; 0.99]$

D

Aseptic

Other

Risk Ratio

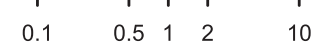

Favors hydrophilic Favors other

M-H, Fixed, $95 \% \mathrm{Cl}$

Trial Events Total Events Total Weight M-H, Fixed, 95\% Cl

Risk Ratio

\begin{tabular}{llllllll}
\hline & & & & & & & \\
King 1992 & 3 & 23 & 5 & 23 & $42.9 \%$ & 0.60 & {$[0.16 ; 2.22]$} \\
Moore 2006 & 9 & 20 & 6 & 16 & $57.1 \%$ & 1.20 & {$[0.54 ; 2.66]$}
\end{tabular}
Moore 2006

$57.1 \% \quad 1.20 \quad[0.54 ; 2.66]$

Total $(95 \% \mathrm{Cl})$

$$
43
$$

39

Total events 12 11

Heterogeneity: $I^{2}=0 \%, \mathrm{~T}^{2}=0, \mathrm{H}=1.00, \mathrm{Q}=0.81, \mathrm{df}=1, p=0.368$

Test for overall effect: $Z=-0.17, p=0.866$

$0.94 \quad[0.48 ; 1.86]$

\begin{tabular}{|c|c|c|c|c|c|c|}
\hline \multirow[b]{2}{*}{ Trial } & \multicolumn{2}{|c|}{ Single } & \multicolumn{2}{|c|}{ Multiple } & \multicolumn{2}{|r|}{ Risk Ratio } \\
\hline & Events & Total & Events & Total & Weight & M-H, Fixed, $95 \% \mathrm{Cl}$ \\
\hline Pachler 1999 & 1 & 32 & 1 & 32 & $14.2 \%$ & $1.00[0.07 ; 15.30]$ \\
\hline Sutherland 1996 & 1 & 16 & 1 & 14 & $15.1 \%$ & $0.88[0.06 ; 12.73]$ \\
\hline King 1992 & 3 & 23 & 5 & 23 & $70.8 \%$ & $0.60 \quad[0.16 ; 2.22]$ \\
\hline Total $(95 \% \mathrm{Cl})$ & & 71 & & 69 & $100.0 \%$ & $0.70[0.24 ; 2.05]$ \\
\hline Total events & 5 & & 7 & & & \\
\hline
\end{tabular}

Heterogeneity: $I^{2}=0 \%, \tau^{2}=0, \mathrm{H}=1.00, \mathrm{Q}=0.15$, df $=2, p=0.930$

Test for overall effect: $Z=-0.66, p=0.512$

$\mathbf{F}$

Hydrophilic Other

Risk Ratio M-H, Fixed, $95 \% \mathrm{Cl}$

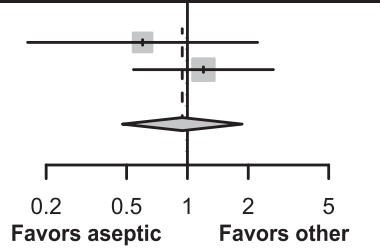

Favors aseptic Favors other

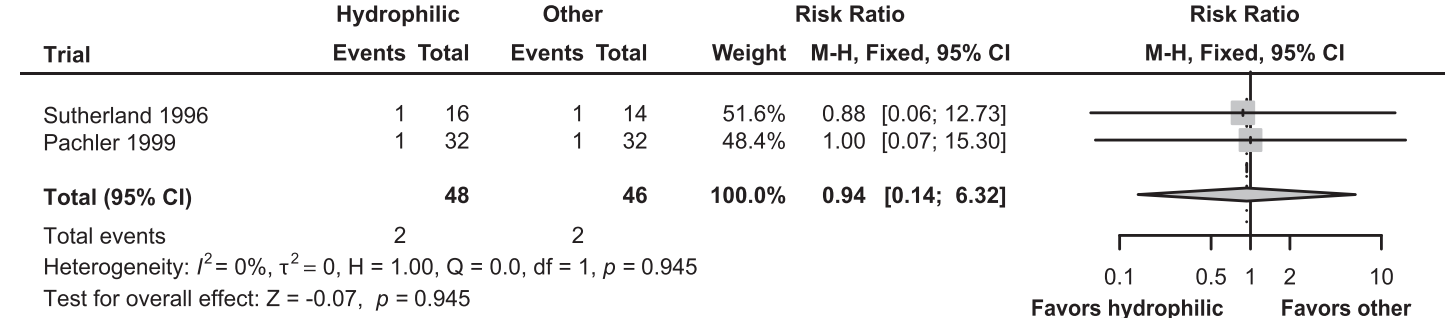

Test for overall effect: $Z=-0.07, p=0.945$ 
and Rehabilitation Research (NIDRR) consensus statement. ${ }^{5}$ Prieto and colleagues also chose to accept definitions for symptomatic UTI as reported in the trials reviewed (p.3). As a result, heterogeneous definitions of symptomatic UTI were included in analysis. However, at the time the Cochrane review was conducted, the Infectious Diseases Society of America (IDSA) 2009 Consensus Statement had already provided the most up-to-date and comprehensive definition of UTI, which specifically covered catheterassociated UTI (Table 2). ${ }^{6}$

\section{Fourth concern (data analysis)}

Of all 39 analyses from the Cochrane review (Supplementary Table 1), 20 consisted of only one trial. However, according to the Cochrane Handbook for Systematic Reviews of Interventions Version 5.1.0 at least two trials are required for meta-analysis. ${ }^{7}$ Of the remaining 19 analyses that compared two or more trials, only four analyses included data that matched the originally published data. There were also inconsistencies with subtotals and totals displayed within the forest plots of numerous analyses. In "Analysis 3.2," Prieto and colleagues "chose not to derive a summary estimate because of heterogeneity amongst the trials and the problem of attrition bias." Surprisingly, although the authors noted the same issue with heterogeneity in "Analysis 2.2" and stated, "We decided not to derive a summary...," they still included subtotal and total summaries in the forest plot of this analysis (pp.73-74).

Following careful re-evaluation of all trials that were included in the Cochrane review, which led to necessary corrections (data selection, extraction, and use of up-to-date definition for UTI), we computed all 19 analyses comprising at least two trials according to the Cochrane Handbook. ${ }^{7}$

In contrast to the review by Prieto and colleagues, we found that "Analysis 2.2" exhibits a trend (albeit small) toward the single use (sterile) of catheters, and "Analysis 3.2" significantly favors hydrophilic catheters (Fig. 1). Further, when we applied the up-to-date (IDSA) definition of symptomatic UTI, at least $50 \%$ of trials from the 2014 Cochrane review "Analysis 2.2 and 3.2" had to be excluded due to outdated UTI definitions (Fig. 1).

\section{Discussion}

Given the presented evidence, we strongly believe that the statement made in the Cochrane review: "...there is still no convincing evidence that the incidence of UTI is affected..." by any of the established intermittent catheterization techniques has to be corrected. When analyses were performed (after data correction) using the 2014 Cochrane review definitions for UTI, no difference was found between single versus multiple use of catheters. However, the use of hydrophilic versus other catheters demonstrated a significantly lower incidence of UTI. Further, when applying the up-to-date IDSA definition of UTI, a trend favoring single versus multiple use of catheters was detected, which is in contrast to the conclusion of Prieto and colleagues. Until evidence can confidently demonstrate that multiple use is as safe as single use of catheters, healthcare providers should advocate a single use of catheters in individuals with SCI, especially considering that catheter cleaning is a major issue because there is no standardized and universally accepted cleaning method that would be the prerequisite for multiple use of catheters. A future and more homogeneous systematic review is necessary to identify evidence that has accumulated since 2014. If analyses remain inconclusive, further high-quality RCTs with adequate number of participants and trial duration, are necessary to derive conclusive results.

\section{Acknowledgments}

Andrei V. Krassioukov had full access to all the data in the study and takes responsibility for the integrity of the data and the accuracy of the data analysis. Study concept and design: Christison, Walter, Wyndaele, Kennelly, Kessler, Krassioukov. Acquisition of data: Christison, Walter, Krassioukov. Analysis and interpretation of data: Christison, Walter, Wyndaele, Kennelly, Kessler, Noonan, Fallah, Krassioukov. Drafting of the manuscript: Christison, Walter, Krassioukov. Critical revision of the manuscript for important intellectual content: Christison, Walter, Wyndaele, Kennelly, Kessler, Noonan, Fallah, Krassioukov. Statistical analysis: Christison, Walter, Noonan, Fallah. Supervision: Krassioukov. Funding: K. Christison is a 2017 University of British Columbia - Faculty of Medicine Summer Student Research Program (FoM SSRP) Award recipient. M. Walter is a 2017 Michael Smith Foundation for Health Research (MSFHR) Research Trainee Award recipient, in partnership with the Rick Hansen Foundation (RHF). The laboratory research of A.V. Krassioukov is supported by funding from the Canadian Foundation for Innovation, BC Knowledge Development Fund, the Canadian Institute for Health Research, Heart and Stroke Foundation Canada, RHF and Institute, Craig Neilsen Foundation, and Wings for Life Foundation.

\section{Author Disclosure Statement}

No competing financial interests exist.

\section{References}

1. Wyndaele, J.J., Brauner, A., Geerlings, S.E., Bela, K., Peter, T., and Bjerklund-Johanson, T.E. (2012). Clean intermittent catheterization and urinary tract infection: review and guide for future research. BJU Int. 110, E910-E917.

2. D'Hondt, F., and Everaert, K. (2011). Urinary tract infections in patients with spinal cord injuries. Curr. Infect. Dis. Rep. 13, 544-551.

3. Prieto, J., Murphy, C.L., Moore, K.N., and Fader, M. (2014). Intermittent catheterisation for long-term bladder management. Cochrane Database Syst. Rev. 9, CD006008.

4. Prieto, J., Murphy, C.L., Moore, K.N., and Fader, M. (2017). WITHDRAWN: Intermittent catheterisation for long-term bladder management. Cochrane Database Syst. Rev. 8, CD006008.

5. NIDRR (1992). The prevention and management of urinary tract infections among people with spinal cord injuries. National Institute on Disability and Rehabilitation Research Consensus Statement. January 27-29, 1992. J Am. Paraplegia Soc. 15, 194-204.

6. Hooton, T.M., Bradley, S.F., Cardenas, D.D., Colgan, R., Geerlings, S.E., Rice, J.C., Saint, S., Schaeffer, A.J., Tambayh, P.A., Tenke, P., and Nicolle, L.E. (2010). Diagnosis, prevention, and treatment of catheter-associated urinary tract infection in adults: 2009 International Clinical Practice Guidelines from the Infectious Diseases Society of America. Clin. Infect. Dis. 50, 625-663.

7. The Cochrane Collaboration. (2011). The Cochrane Handbook for Systematic Reviews of Interventions, Version 5.1.0. J.P.T. Higgins, and S. Green (eds). Available at http://handbook.cochrane.org. Last accessed January 10, 2018.

Address correspondence to:

Andrei V. Krassioukov, MD, PhD, FRCPC

International Collaboration on Repair Discoveries (ICORD) 818 West 10th Avenue

Vancouver, British Columbia V5Z 1 M9

Canada

E-mail: krassioukov@icord.org 